\title{
The Effectiveness and Value of Treatments for Spinal Muscular Atrophy
}

\section{A Summary from the Institute for Clinical and Economic Review's New England Comparative Effectiveness Public Advisory Council}

\author{
Steven D. Pearson, MD, MSc; Praveen Thokala, PhD, MASc; \\ Matt Stevenson, PhD, BSc; and David Rind, MD, MSc
}

T here are 2 treatments approved by the U.S. Food and Drug Administration (FDA) for spinal muscular atrophy (SMA), a rare genetic neuromuscular disease affecting approximately 1 in 10,000 live births in the United States. Spinraza (nusinersen, Biogen Idec), an antisense oligonucleotide, was approved in 2016 for the treatment of any subtype of SMA and targets SMN2 to create more functional SMN protein. It is administered via intrathecal injection, with 4 loading doses (day 0, day 14, day 28, and day 63) and maintenance doses every 4 months thereafter. Zolgensma (onasemnogene abeparvovec, Novartis AG/AveXis) was approved on May 24, 2019, to treat patients aged less than 2 years with any subtype of SMA. Zolgensma is a gene therapy administered in a single intravenous dose that uses the adeno-associated virus serotype 9 vector (AAV9) to deliver a copy of the SMN1 gene to replace the native defective or absent gene.

In this article, we present a summary of the systematic literature review and cost-effectiveness analysis of Sprinraza and Zolgensma performed by our research staff at the Institute for Clinical and Economic Review (ICER). We also present the highlights of the deliberation on this evidence review and a subsequent policy discussion. In addition, this article includes information contained in an update to the ICER report posted on May 24, 2019, that provided a synopsis of new evidence that had emerged after the ICER meeting and immediately before the FDA approval of Zolgensma. The full updated report is available on the ICER website at https://icer-review.org/material/sma-finalevidence-report/.

\section{Summary of Findings}

\section{Clinical Effectiveness}

Spinraza: SMA Type I. For a treatment targeting a serious ultra-rare condition, there is a relatively robust evidence base on Spinraza. Overall, we identified 4 clinical trials, including 2 randomized controlled trials (RCTs; ENDEAR and EMBRACE) ${ }^{1,2} 1$ open-label dose-escalation study (CS3A), ${ }^{3}$ and 1 open-label extension (SHINE). ${ }^{4}$ We also included 3 openlabel extended access program (EAP) studies..$^{5-7}$

J Manag Care Spec Pharm. 2019;25(12):1300-06

Copyright $\odot 2019$, Academy of Managed Care Pharmacy. All rights reserved.
For Type I SMA, results were available from both RCTs and the dose-escalation study. Longer-term results were also available for infants in ENDEAR who enrolled in the open-label extension study. ${ }^{8}$ In all studies, Spinraza demonstrated statistically significant reductions in the need for ventilatory support and improvements in survival. For example, in the ENDEAR study, Spinraza demonstrated a $47 \%$ decrease in the risk of death or permanent assisted ventilation (HR [95\% CI]: 0.53 [0.32-0.89], $P=0.005$ ). ${ }^{1}$ Spinraza was also superior to standard of care in improving motor function and milestone achievement. Although Spinraza is not a cure, among infants with at least 6 months of follow-up in ENDEAR, no infant who received sham achieved any motor milestone, whereas $22 \%$ of patients who received Spinraza achieved head control, and $1 \%$ achieved standing with assistance. Long-term follow-up data show additional motor milestone achievements for infants receiving Spinraza who transitioned from ENDEAR to the open-label extension study. In data from an interim analysis (June 15, 2017), after 576 days, approximately $45 \%$ of infants achieved full head control, and $29 \%$ were able to sit independently.

Spinraza: SMA Types II and III. For later-onset SMA, there was 1 RCT (CHERISH) of Spinraza versus sham control in children aged 2-12 years, and 1 phase Ib/Ila open-label study (CS2/CS12) in children aged 2 through 15 years. ${ }^{9,10}$ In addition, the sham-controlled EMBRACE trial, which included children with Type I, II, or III, presented results on a subgroup of children diagnosed with later-onset SMA. ${ }^{11}$

In CHERISH, Spinraza demonstrated statistically significant improvements in changes from baseline motor function versus the sham control, but new achievements in walking with assistance, standing alone, and any motor milestone were similar between Spinraza and sham control groups. There were no deaths during CHERISH or CS2/CS12, and no data on permanent ventilation were available.

Spinraza: Presymptomatic SMA. One ongoing, single-arm study (NURTURE) reported on Spinraza treatment in 25 presymptomatic infants with 2 or 3 copies of SMN2. ${ }^{12,13}$ As of May 2018, all 25 children were alive, and none required permanent ventilatory support. However, many children with 1 year of follow-up had developed 1 or more clinical symptoms of SMA. Four (16\%) children met the primary outcome of requiring 
nonpermanent respiratory intervention ( 6 or more hours per day for 7 consecutive days or tracheostomy). All of these children received respiratory intervention during an acute, reversible illness, and none required tracheostomy. With a median time on treatment of 27.1 months, the mean motor function scores reflected near-maximal function. Caregivers reported that all 25 children had achieved sitting without support; 22 of 25 (88\%) children had achieved walking with assistance; and 17 of 25 (68\%) had achieved walking alone.

Zolgensma: SMA Type I. At the time of the original ICER report, data were available only from a single, small (12 patients) open-label, 2-cohort clinical trial (CL-101) of Zolgensma and its extension study (START) in Type I SMA. ${ }^{14}$ All infants treated with Zolgensma in CL-101 were alive and event-free through 24 months of follow-up. Improvements in motor function scores were observed among treated infants, with $92 \%$ of patients achieving head control and $17 \%$ able to walk independently. Two more children achieved standing with support during additional follow-up in START. ${ }^{15}$

In the update to the ICER report, we included additional data from ongoing trials of Zolgensma that were presented at conferences in April and May 2019.16-18 In a phase III, single-arm trial (STRlVE) of infants with Type I SMA, 21 of 22 infants treated with Zolgensma were alive, with a median age of 14.4 months. The single death was deemed not related to treatment. Five months after treatment, motor function scores increased by an average of 14.3 points, which was similar to the results from the earlier START trial.

Zolgensma: SMA Types II and III. As noted earlier, at the time of the original ICER report, there were no data reported from trials of Zolgensma in presymptomatic SMA or in SMA Types II and III. Early interim data presented in April and May 2019 included results from a phase I dose comparison trial (STRONG) of Zolgensma in patients with Type II SMA. Early results from this trial showed that the treatment was well tolerated and a number of the patients achieved new motor milestones. ${ }^{18}$

Zolgensma: Presymptomatic SMA. In the update to the ICER report, we summarized conference data from a phase III singlearm trial (SPR1NT) that evaluated Zolgensma in presymptomatic patients with 2 or 3 copies of SMN2. ${ }^{16}$ Patients had been diagnosed near the time of birth and were 6 weeks of age or younger at the time of treatment. After a median follow-up of 5.4 months (median age 6.1 months), all 18 children were alive and "event free." Among 8 patients with 2 copies of SMN2 (presumed Type I SMA), all reportedly achieved age-appropriate motor milestones, including 4 who could sit without support and 1 who could stand with assistance.

\section{Limitations of the Clinical Evidence}

All trials available at the time of this review showed prolonged survival and improved motor function compared with historical controls or sham injections for Spinraza (SMA Types I-III) and Zolgensma (SMA Type I). However, even after the update to the original ICER report, there remain several important uncertainties. First, for both interventions, the narrow eligibility criteria of trials and the limited sample sizes (especially for Zolgensma) raise concerns about generalizability of results to the wider population of patients with SMA. In particular, data are not available to evaluate the effect of treatment on patients who have been so severely affected by SMA that they already require permanent ventilation and/or who have developed scoliosis, which may limit the benefits of improved respiratory muscle function.

The evidence on Spinraza, particularly in Types II and III and presymptomatic patients, is more robust than the early evidence on Zolgensma and provides more certainty about its intermediate-term effectiveness. Currently available data do not suggest diminishing benefit over time, but for both treatments the longer-term durability and magnitude of benefit and potential risks remain uncertain. Spinraza prescribing information notes the risks of thrombocytopenia and renal toxicity, and clinical experts have mentioned concerns about the possible negative effects of long-term repeated lumbar punctures.

For Zolgensma, there remains data on only a very small number of patients for a relatively short amount of time. If the therapeutic gene expression wanes over time, the effect on longterm outcomes is uncertain. It is also possible that antibodies to the viral vector may be generated, eliminating the possibility of repeat treatment with Zolgensma. In addition, without randomized trial treatment effects to judge against, historical controls can exaggerate perceived treatment effects. For example, in older natural history studies, approximately $68 \%$ of patients with Type I SMA died by 2 years of age. ${ }^{19,20}$ In part, due to the improvements in and increased utilization of nutritional and respiratory support, more recent estimates of mortality are approximately 30\% at 2 years of age with approximately half of survivors reliant on noninvasive ventilation. ${ }^{21}$

Given the differences in baseline characteristics between the trials of Type I SMA, direct comparisons of relative effectiveness between Zolgensma and Spinraza should be avoided. For example, there are differences in age at treatment initiation and duration of disease, which are known to be modifiers of treatment effect. In addition, the time point of primary outcome analysis and approach for assessing motor milestones differ between the studies on Spinraza and Zolgensma.

For presymptomatic patients, very early results suggest that treatment with Spinraza or Zolgensma may provide more benefit to patients than waiting until symptoms have developed. This would be consistent with the understanding of the pathophysiology of the condition, in which motor neurons are lost in an ongoing, cumulative fashion. Whether there are greater risks for treatment of very young children remains highly uncertain.

Finally, it should be noted that there are no data on concurrent or sequential use of Spinraza and Zolgensma. Some patients who 


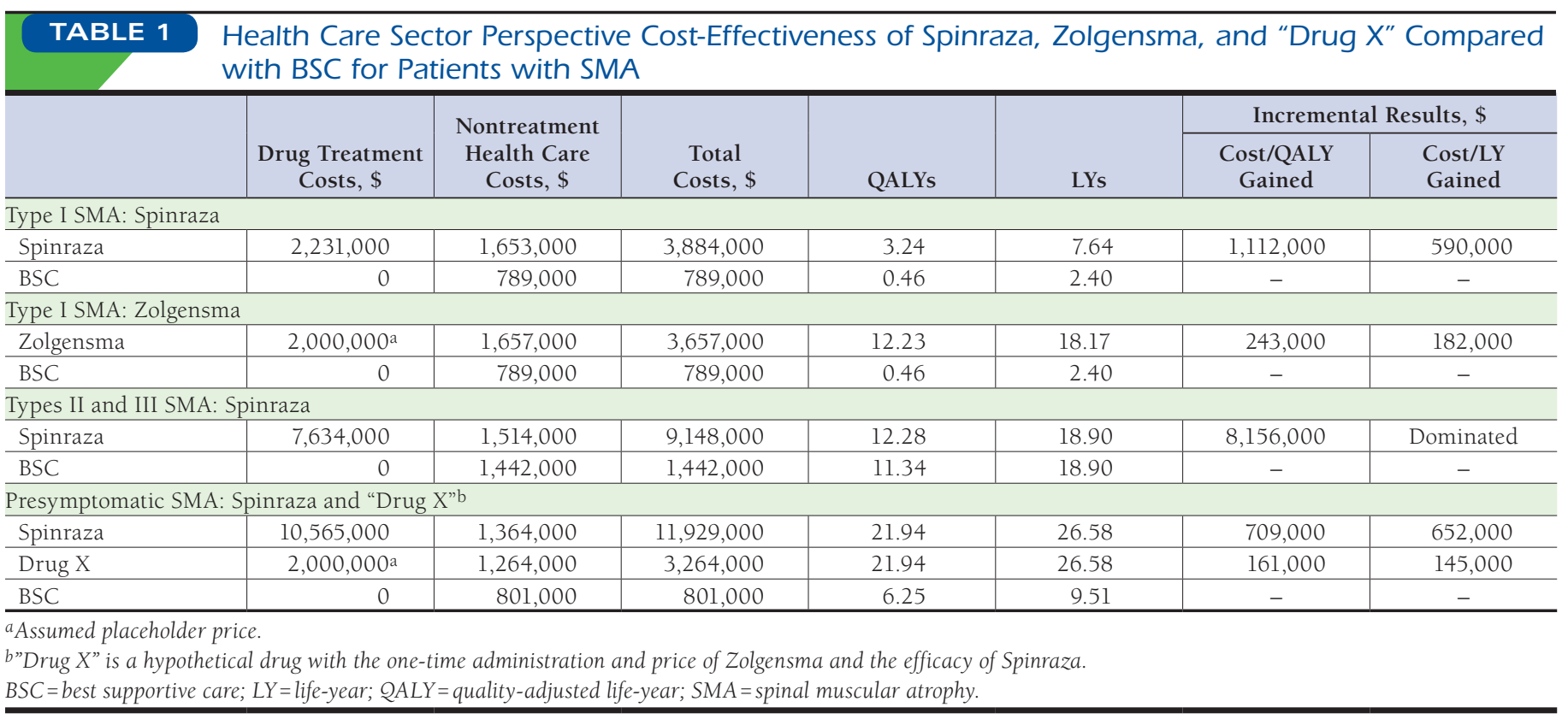

received Zolgensma in START went on to take Spinraza after the trial, but the reason these children were treated with both agents and the outcomes of this treatment sequence are unknown.

\section{Long-Term Cost-Effectiveness}

We developed separate de novo economic models for SMA Type I, Types II and III, and presymptomatic. Spinraza was compared with best supportive care (BSC) in each model, whereas Zolgensma was evaluated against BSC solely in Type I SMA. For each population, we estimated the lifetime costs, life-years (LYs) gained, and quality-adjusted life-years (QALYs) gained, discounted at 3\% per annum, for Spinraza and BSC.

The models were dependent on 3 constructs: motor function milestones achieved, need for permanent ventilation, and the time to death. All 3 models used the same structure and contained 2 main components: a short-term model concordant with clinical study data and a long-term extrapolation model. For Zolgensma, since at the time of modeling there was no announced price, we used a placeholder one-time cost of $\$ 2$ million for the base-case analysis. Full details of each model, including all assumptions, sources for utilities and costs, and the analyses conducted, are available on ICER's website.

In addition to the models previously described, we included in our report an analysis of a hypothetical "Drug X" for presymptomatic SMA, assumed to have the one-time administration and pricing structure of Zolgensma and the efficacy of Spinraza (used as a placeholder in the absence of data on Zolgensma). We chose to present this model at the time due to our understanding that data on Zolgensma for presymptomatic SMA would be forthcoming, and we felt it important to provide a context for policymakers to consider the potential cost-effectiveness of a onetime treatment for presymptomatic SMA. As it turned out, data on Zolgensma for presymptomatic SMA were announced in April and May 2019, and the FDA approval for Zolgensma included an indication for use in this population. The clinical data are still preliminary and have not been subject to peer review, but policymakers may wish to consider the results from ICER's modeling of Drug X in thinking about the value of Zolgensma.

Results from the health care sector perspective for the 3 different populations are presented in Table 1. For the Type I SMA model, incremental cost-effectiveness ratios compared with BSC are approximately $\$ 1.1$ million per QALY gained for Spinraza and approximately $\$ 243,000$ per QALY for Zolgensma. For SMA Types II and III, the incremental cost-effectiveness ratio of Spinraza compared with BSC is approximately $\$ 8$ million per QALY gained.

Cost-effectiveness of SMA treatment was found to be best for patients with presymptomatic SMA. In the presymptomatic model, the incremental cost-effectiveness ratio of Spinraza compared with BSC is approximately $\$ 700,000$ per QALY. For Drug X, the incremental cost-effectiveness ratio was $\$ 161,000$ per QALY gained, and $\$ 145,000$ per LYs gained, close to the upper bound of traditional cost-effectiveness ranges in the United States.

Our cost-effectiveness analyses have several limitations. As noted earlier, there are no long-term follow-up data available on either Spinraza or Zolgensma, resulting in considerable uncertainty related to the long-term outcomes of treated patients. This uncertainty in long-term survival was partially addressed through multiple sensitivity and scenario analyses presented in the full report. Furthermore, minor gradations of improved 
When compared with supportive care alone, does the addition of Spinraza or Zolgensma offer 1 or more of the following "other benefits"?

\begin{tabular}{|c|c|c|}
\hline \multirow[b]{2}{*}{ Potential Benefit } & \multicolumn{2}{|c|}{ Panel Votes } \\
\hline & Spinraza & Zolgensma \\
\hline $\begin{array}{l}\text { This therapy offers reduced complexity compared with other treatment options that will improve patient outcomes } \\
\text { in the real world. }\end{array}$ & N/A & $12 / 12$ \\
\hline $\begin{array}{l}\text { This therapy has a different mechanism of action or approach that will allow successful treatment of many patients } \\
\text { for whom other available treatments have failed. }\end{array}$ & N/A & N/A \\
\hline This therapy will significantly reduce caregiver or broader family burden. & $12 / 12$ & $11 / 12$ \\
\hline $\begin{array}{l}\text { This therapy will have a significant effect on improving patients'/caregivers' ability to return to work and/or their } \\
\text { overall productivity. }\end{array}$ & $10 / 12$ & $10 / 12$ \\
\hline $\begin{array}{l}\text { This therapy will have a significant effect on the entire "infrastructure" of care, including effects on screening for } \\
\text { affected patients, on the sensitization of clinicians, and on the dissemination of understanding about the condition, } \\
\text { that may revolutionize how patients are cared for in many ways that extend beyond the treatment itself. }\end{array}$ & $12 / 12$ & $11 / 12$ \\
\hline $\begin{array}{l}\text { There are other important benefits—or disadvantages—that should have an important role in judgments of the } \\
\text { value of this therapy. }\end{array}$ & N/A & N/A \\
\hline
\end{tabular}

muscle function that may not be captured in existing outcome measures can have real effects on patient mobility and quality of life. For example, with modern technology, the ability to move just a single finger can enhance independence and quality of life by allowing a patient to operate an iPad or move the joystick of a motorized wheelchair. In part, because we were aware of this limitation in the recorded outcomes, we added an extra utility benefit in the treatment arms compared with BSC to make allowances for better functioning within broad health states.

\section{Policy Discussion}

The New England Comparative Effectiveness Public Advisory Council (New England CEPAC) is one of the independent appraisal committees convened by our institute to engage in the public deliberation of the evidence on clinical and costeffectiveness of health care interventions.

The New England CEPAC deliberated on ICER's report on Spinraza and Zolgensma at a public meeting on March 7, 2019. Following discussion, the CEPAC panel members first voted unanimously that the evidence was adequate to demonstrate the superiority of both Spinraza and Zolgensma to BSC in Type I SMA and, for Spinraza, in Types II and III SMA, as well. Voting was 10-2 that the evidence was adequate to demonstrate superiority in presymptomatic SMA for Spinraza. No votes were taken on Zolgensma other than for its use in Type 1 SMA.

The CEPAC panel also voted on "other potential benefits" and "contextual considerations" as part of a process intended to signal to policymakers whether there are important considerations when making judgments about long-term value for money not adequately captured in analyses of clinical and/or cost-effectiveness. The results in Table 2 and Table 3 highlight several factors that the CEPAC panel felt were particularly important for judgments of value, including the effect on caregiver burden and the fact that these treatments are the first for this very severe condition.
Zolgensma was noted by all panel members as having a reduced complexity (i.e., no need for ongoing lumbar punctures) compared with Spinraza that could improve patient outcomes in real-world practice.

The culminating vote of the CEPAC was on the "long-term value for money" of Spinraza and Zolgensma. Despite the strong affirmation of the clinical and broader benefits of Spinraza, the CEPAC voted unanimously that at current pricing it represented a low long-term value for money in any subset of SMA. No vote on the value of Zolgensma was taken at this meeting, given that no price for Zolgensma had yet been announced.

The culminating policy roundtable discussion explored how best to translate the evidence and broader perspectives discussed into clinical practice and into pricing and insurance coverage policies. The full set of resulting policy recommendations can be found in the final evidence report. Selected key perspectives on potential coverage policy considerations are described below.

1. Payers should provide responses to prior authorization requests within 48 hours. Diagnosis of SMA in an infant should be treated by providers and payers as an emergency requiring rapid decision making and the delivery of treatment as soon as possible. Payers should develop fail-safe mechanisms to ensure that these requests are evaluated and responded to within 48 hours. Payers should make every attempt to communicate with providers and families to resolve any prior authorization challenges as soon as possible.

2. Prior authorization criteria should reflect evolving evidence and clinical expert input.

a. Diagnosis: Insurers should not require repeated documentation of genetic testing results. Given that screening at birth will soon become universal, presymptomatic individuals with different numbers of SMN2 copies will be identified. Although genotype is not precisely predictive of phenotype, existing research suggests that a 


\begin{tabular}{|c|c|c|}
\hline \multirow[b]{2}{*}{ Contextual Consideration } & \multicolumn{2}{|c|}{ Panel Votes } \\
\hline & Spinraza & Zolgensma \\
\hline $\begin{array}{l}\text { This therapy is intended for the care of individuals with a condition of particularly high severity in terms of effect } \\
\text { on length of life and/or quality of life. }\end{array}$ & $11 / 12$ & $10 / 12$ \\
\hline $\begin{array}{l}\text { This therapy is intended for the care of individuals with a condition that represents a particularly high lifetime bur- } \\
\text { den of illness. }\end{array}$ & $11 / 12$ & $10 / 12$ \\
\hline This therapy was the first to offer any improvement for patients with this condition. & $12 / 12$ & Not asked \\
\hline $\begin{array}{l}\text { Compared with best supportive care, there is significant uncertainty about the long-term risk of serious side effects } \\
\text { of this therapy. }\end{array}$ & $7 / 12$ & $6 / 12$ \\
\hline $\begin{array}{l}\text { Compared with best supportive care, there is significant uncertainty about the magnitude or durability of the } \\
\text { long-term benefits of this therapy. }\end{array}$ & $7 / 12$ & $7 / 12$ \\
\hline $\begin{array}{l}\text { There are additional contextual considerations that should have an important role in judgments of the value of this } \\
\text { therapy. }\end{array}$ & N/A & N/A \\
\hline
\end{tabular}

N/A= not applicable.

very small number of individuals with 4 or more copies of SMN2 will develop the most severe forms of SMA. A recent article authored by clinical experts from across the United States, including many with research and other links to industry, found divided opinions on whether individuals found at birth to have 4 or more copies of SMN2 should be treated immediately or whether it was reasonable to wait and monitor them to see if any signs of diminished muscle function emerged. The final proposal from this group supported the option of surveillance with the possibility of later treatment for this subpopulation. ${ }^{22}$

b. Age: For symptomatic patients, based on the lack of data on treatment among older patients, some countries have limited coverage to patients under the age of 12 or 15 years, but patient and clinical expert testimony suggests that there is no basis for assuming that benefits cannot be significant for patients with Types II and III at all ages.

c. Other clinical criteria: For symptomatic patients, payers may opt to have no clinical criteria related to severity or they may consider the option of requiring that clinical criteria be met that demonstrate that the patient is not too severely affected in some way to retain the possibility of benefit from treatment. For example, some payers have required that patients not be on permanent ventilation. Although there are no data on the benefits of initiating Spinraza treatment among permanently ventilated patients, family and clinical expert testimony argued that ventilated patients can benefit from treatment even with relatively small improvements in motor function that can allow the self-direction of motorized wheelchairs or the use of tablets for communication.

Some countries have not provided coverage for Spinraza when patients have attained the ability to walk independently. Although the cost-effectiveness of treat- ment for symptomatic patients is worse among patients who are less severely affected, clinical experts and patient representatives argued that for some patients who can walk independently there are still important upper limb motor function benefits that are possible with treatment.

d. Renewal criteria: Many payers will seek to set a time threshold at which coverage must be reassessed in light of whether there have been demonstrated benefits of treatment. Although a clear threshold is not evident from trial data, clinical experts advised that it is not unreasonable to expect results after 6-12 months of treatment. If there has been no improvement, or at least no halt to a steady decline in symptoms at that time, payers may determine that continued coverage for Spinraza is not medically necessary. Of note, some countries have used achievement or maintenance of sitting as a single outcome measure by which to determine whether continued use of Spinraza is justified, but clinical expert comment suggested that for many patients sitting is not a relevant measure of clinical benefit. Alternatively, given the clinical heterogeneity of patients, and the challenge of determining which clinical outcome measure is best suited for a specific patient, payers may opt for clinician attestation as the most reasonable option for determining whether coverage should be renewed.

\section{Conclusions}

There is no more exciting development in medicine than the introduction of new, highly effective treatments for a condition that affects children and leads to substantial disability and death. The evidence on Spinraza and Zolgensma is still early and, especially for Zolgensma, remains highly uncertain in terms of true long-term outcomes. Nonetheless, the short-term benefits demonstrated in the most severe form of SMA have been substantial, and it is likely that use of both agents will 
shift toward use in presymptomatic patients as genetic screening at birth becomes more widespread.

Despite the clinical benefits seen in early studies, economic modeling in which these early benefits of treatment are assumed to be durable throughout patients' lifetimes finds that the incremental cost-effectiveness ratio for Spinraza, at its current pricing, is far beyond the usual boundaries considered cost-effective for the U.S. health care system. Zolgensma, however, as a one-time therapy, gains a cost-effectiveness advantage over a treatment that must be administered in a chronic fashion throughout the lifetime of the patient. Our economic modeling of Drug X for presymptomatic SMA can serve as a surrogate for Zolgensma, and with the early data for Zolgensma in this patient group now appearing, we believe that Zolgensma's announced price of \$2.1 million lies within the highest part of a "fair" cost-effectiveness range. It must be remembered that the evidence on Zolgensma is early, has not yet appeared in peer-reviewed journals, and its announced price would not be deemed cost-effective for treatment of the current prevalent SMA population. Further research and efforts to link the price of these new treatments for SMA to their demonstrated long-term benefits for patients will require concerted collaborative efforts among manufacturers, payers, patients, and clinicians.

\section{Authors}

STEVEN D. PEARSON, MD, MSc, and DAVID RIND, MD, MSC, Institute for Clinical and Economic Review, Boston, Massachusetts. PRAVEEN THOKALA, PhD, MASc, and MATT STEVENSON, PhD, BSc, Health Economics and Decision Science, School of Health and Related Research, The University of Sheffield, South Yorkshire, England.

AUTHOR CORRESPONDENCE: Steven D. Pearson, MD, MSc, President, Institute for Clinical and Economic Review, Two Liberty Square, 9th Fl., Boston, MA 02109. E-mail: spearson@icer-review.org.

\section{DISCLOSURES}

Funding for this summary was contributed by the Laura and John Arnold Foundation and California Health Care Foundation to the Institute for Clinical and Economic Review (ICER), an independent organization that evaluates the evidence on the value of health care interventions. ICER's annual policy summit is supported by dues from Aetna, AHIP, Anthem, Blue Shield of California, CVS Caremark, Express Scripts, Harvard Pilgrim Health Care, Cambia Health Solutions and MedSavvy, United Healthcare, Kaiser Permanente, Premera Blue Cross, AstraZeneca, Genentech, GlaxoSmithKline, Johnson \& Johnson, Merck, National Pharmaceutical Council, Sanofi, Alnylam, Novartis, HealthPartners, Blue Cross Blue Shield of Massachusetts, Health Care Services Corporation, Mallinkrodt Pharmaceuticals, Prime Therapeutics, Regeneron, National Institute for Health Care Management, Commonwealth Fund, Partners Healthcare, New England States Consortium Systems, Allergan, Biogen, Editas, LEO Pharma, and HealthFirst. ICER has also received grants from Kaiser Foundation Health Plan, California Health Care Foundation, and the Laura and John Arnold Foundation. Pearson and Rind are employees of ICER. Thokala and Stevenson have no potential conflicts of interest to disclose.

\section{REFERENCES}

1. Finkel RS, Mercuri E, Darras BT, et al. Nusinersen versus sham control in infantile-onset spinal muscular atrophy. N Engl J Med. 2017;377(18):1723-32.

2. ClinicalTrials.gov. Identifier NCT02462759. A study to assess the safety and tolerability of nusinersen (ISIS 396443) in participants with spinal muscular atrophy (SMA). (EMBRACE). September 25, 2018. Available at: https://www. clinicaltrials.gov/ct2/show/NCT02462759. Accessed November 1, 2019

3. Finkel RS, Chiriboga CA, Vajsar J, et al. Treatment of infantile-onset spinal muscular atrophy with nusinersen: a phase 2, open-label, dose-escalation study. Lancet. 2016;388(10063):3017-26.

4. Castro D, Farrar M, Finkel R, et al. Interim report on the safety and efficacy of longer-term treatment with nusinersen in infantile-onset spinal muscular atrophy: results from the SHINE study. Neuromuscul Disord. 2018;28:S79-S80

5. Pechmann A, Langer T, Schorling D, et al. Evaluation of children with SMA type 1 under treatment with nusinersen within the expanded access program in Germany. J Neuromuscul Dis. 2018;5(2):135-43.

6. Pane M, Palermo C, Messina S, et al. Nusinersen in type 1 SMA infants, children and young adults: preliminary results on motor function. Neuromuscul Disord. 2018;28(7):582-85.

7. Farrar MA, Teoh HL, Carey KA, et al. Nusinersen for SMA: expanded access programme. J Neurol Neurosurg Psychiatry. 2018;89(9):937-42

8. Haataja L, Mercuri E, Regev R, et al. Optimality score for the neurologic examination of the infant at 12 and 18 months of age. J Pediatr. 1999;135 (2 Pt 1):153-61.

9. Mercuri E, Darras BT, Chiriboga CA, et al. Nusinersen versus sham control in later-onset spinal muscular atrophy. N Engl J Med. 2018;378(7):625-35.

10. Chiriboga C, Darras B, Montes J. Nusinersen in treatment-naive children with later-onset spinal muscular atrophy (SMA): efficacy results from a phase 1b/2a multicenter study (CS2) and its open-label extension (CS12). Paper presented at: 2017 Annual Spinal Muscular Atrophy Conference; June 29-July 2, 2017; Orlando, FL.

11. Shieh PB, Acsadi G, Mueller-Felber W, et al. B.06 Safety and efficacy of nusinersen in infants/children with spinal muscular atrophy (SMA): part 1 of the phase 2 EMBRACE study [abstract]. Can J Neurol Sci. 2018;45(s2):S13. Available at: https://doi.org/10.1017/cjn.2018.94. Accessed November 1, 2019.

12. De Vivo D, Bertini E, Hwu WL, et al. One-year outcomes following treatment with nusinersen: interim results from the NURTURE study of presymptomatic infants with genetically diagnosed spinal muscular atrophy (SMA) [abstract]. Ann Neurol. 2017;82(S21):S265-S266

13. De Vivo DC, Bertini E, Hwu W, et al. B.05 Nusinersen in infants who initiate treatment in a presymptomatic stage of spinal muscular atrophy (SMA): interim results from the Phase 2 NURTURE study [abstract]. Can J Neurol Sci. 2018;45(s2):S12-S13. Available at: https://doi.org/10.1017/ cjn.2018.93. Accessed November 1, 2019.

14. Mendell JR, Al-Zaidy S, Shell R, et al. Single-dose gene-replacement therapy for spinal muscular atrophy. N Engl J Med. 2017;377(18):1713-22.

15. Shell R, Al-Zaidy S, Arnold W, et al. P.178 AVXS-101 phase 1 gene therapy clinical trial in spinal muscular atrophy type 1: improvement in respiratory and bulbar function reduces frequency and duration of hospitalizations compared to natural history [abstract]. Neuromuscul Disord. 2018;28 (Suppl 2):S82. Available at: https://www.nmd-journal.com/article/S09608966(18)30769-7/fulltext. Accessed November 1, 2019.

16. Day J, Chiriboga C, Crawford T, et al. AVXS-101 gene-replacement therapy (GRT) for spinal muscular atrophy type 1 (SMA1): pivotal phase 3 study (STRlVE) update. Presented at: AAN 2019 Annual Meeting; May 4-10, 2019; Philadelphia, PA.

17. Schultz M, Swoboda K, Farrar M, et al. AVXS-101 gene-replacement therapy (GRT) in presymptomatic spinal muscular atrophy (SMA): study update. Presented at: AAN 2019 Annual Meeting; May 4-10, 2019; Philadephia, PA. 
18. Finkel R, Day J, Darras B, et al. Phase 1 study of intrathecal administration of AVXS-101 gene-replacement therapy (GRT) for spinal muscular atrophy type 2 (SMA2) (STRONG). Presented at: AAN 2019 Annual Meeting; May 4-10, 2019; Philadelphia, PA.

19. Mannaa MM, Kalra M, Wong B, Cohen AP, Amin RS. Survival probabilities of patients with childhood spinal muscle atrophy. J Clin Neuromuscul Dis. 2009;10(3):85-89.
20. Oskoui M, Levy G, Garland CJ, et al. The changing natural history of spinal muscular atrophy type 1. Neurology. 2007;69(20):1931-36.

21. Finkel RS, McDermott MP, Kaufmann P, et al. Observational study of spinal muscular atrophy type I and implications for clinical trials. Neurology. 2014;83(9):810-17.

22. Glascock J, Sampson J, Haidet-Phillips A, et al. Treatment algorithm for infants diagnosed with spinal muscular atrophy through newborn screening. J Neuromuscul Dis. 2018;5(2):145-58.

\title{
Spinal Muscular Atrophy Therapies: ICER Grounds the Price to Value Conversation in Facts
}

\author{
Catherine I. Starner, PharmD, and Patrick P. Gleason, PharmD
}

\section{COMMENTARY} for Clinical and Economic Review (ICER) released its final evidence report on the effectiveness and value of spinal muscular atrophy (SMA) treatments. ${ }^{1}$ The 2 SMA treatments approved by the U.S Food and Drug Administration were the focus of the report. Spinraza (nusinersen) is a survival motor neuron-2-directed antisense oligonucleotide indicated for SMA in pediatric and adult patients. ${ }^{2}$ Zolgensma (onasemnogene abeparvovec-xioi) is a 1-time gene therapy infusion indicated for SMA in children aged $<2$ years with bi-allelic mutations in the survival motor neuron 1 (i.e., SMA type 1) gene. ${ }^{3}$ Zolgensma has been dubbed the "most expensive drug in the world" at $\$ 2.125$ million. ${ }^{4}$ The ICER quality-adjusted life-year (QALY) costeffective model for treating SMA type 1 infants with symptoms who are $<8$ months found that Zolgensma should be priced at $\$ 310,000$ for a $\$ 100,000$ investment per QALY gained, 6.9-fold

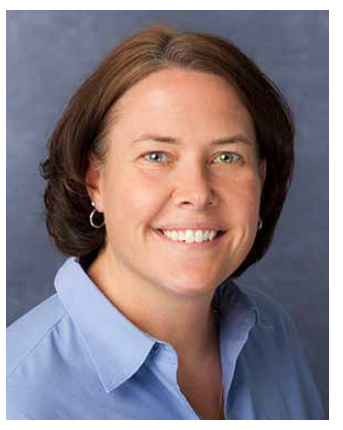
investment point of $\$ 100,000$ per QALY. Therefore, the Spinraza current price should be discounted by 10 -fold for a fair price at an investment point of $\$ 100,000$ per QALY gained.

Why are the cost-effective numbers so different for Spinraza and Zolgensma? The Spinraza value price is maximally $\$ 36,400$ a year due to the limited clinical, yet statistically significant, effectiveness. In the Spinraza infant onset type 1 SMA ENDEAR study, ${ }^{5}$ no infant who received sham therapy achieved any development milestone; how-

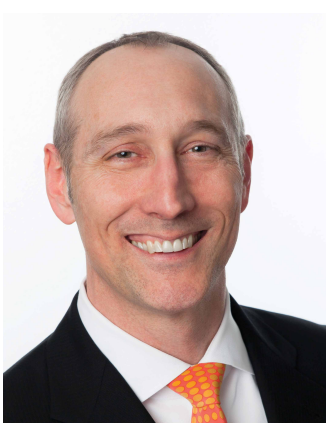
ever, 22\% of Spinraza-treated infants achieved head control, and $1 \%$ achieved standing with assistance. After 576 days of follow-up in the SHINE study, ${ }^{6}$ approximately $45 \%$ of treated infants achieved full head control, and 29\% achieved sitting independently. The median time to death or permanent ventilation was 22.6 weeks in the sham control group and 73.0 weeks in the Spinraza group. With Zolgensma, at 24 months, $92 \%$ of infants achieved head control, and $17 \%$ could walk independently. According to the ICER report, "By May 2018, careless than list price. When the ICER cost-effective models are run for infants with SMA type 1 who are presymptomatic (i.e., at or near birth), the Zolgensma price at $\$ 100,000$ per QALY is $\$ 1.1$ million, 1.9-fold lower than list price. Spinraza is an intermittent intrathecal infusion with a wholesale acquisition cost of $\$ 805,000$ for the first year of therapy and $\$ 380,000$ per year thereafter. The Spinraza SMA type 1 presymptomatic costeffective modeling found a price of $\$ 72,800$ per QALY in year 1 and $\$ 36,400$ per QALY gained in year 2 and beyond, at the givers reported all 25 (100\%) had achieved sitting without support, 22/25 (85\%) of children had achieved walking with assistance, and 17/25 (68\%) had achieved walking alone."' Among the 25 Zolgensma-treated patients, there has been 1 nontreatment-related death and another potentially treatmentrelated death. ${ }^{7}$ Finally, the ICER analysis found higher gains in QALY and life-years gained for Zolgensma compared with Spinraza. ${ }^{1}$ Many of the additional Spinraza life-years gained could be spent requiring caregiving and medical care, resulting in the Spinraza maximum price of $\$ 36,400$ per year and the Zolgensma one-time price of $\$ 1.1$ million for SMA type 1 presymptomatic treatment. ${ }^{1}$ 